\title{
RESISTENCIA DE UNIÓN A LA DENTINA DE DOS CEMENTOS SELLADORES DE ENDODONCIA DESPUÉS DE LA MEDICACIÓN INTRACONDUCTO DE HIDRÓXIDO DE CALCIO CON DOS TIPOS DE VEHÍCULOS
}

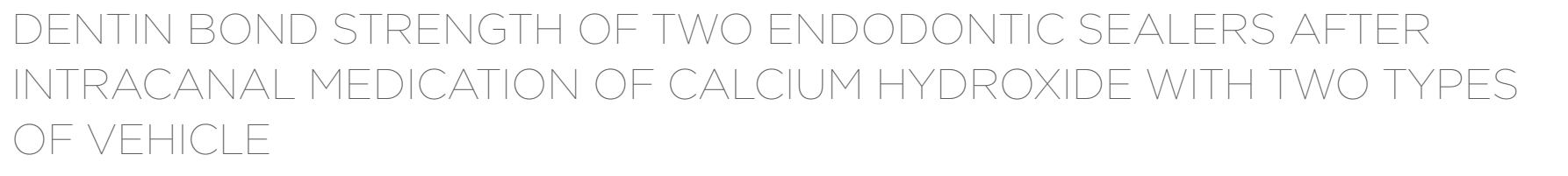

\section{Angela Carolina Reinoso-Trabucco * (1) \\ angela.reinoso.dentist@gmail.com}

Carmen Rosa García-Rupaya ${ }^{2}$ cgarciar@ucientifica.edu.pe

Artículo recibido: 04/05/2020

Arbitrado por pares

Artículo aceptado: 18/06/2020

Artículo publicado: 24/08/2020

\section{* Autor corresponsal:}

Angela Carolina Reinoso-Trabucco angela.reinoso.dentist@gmail.com

Citar como: Reinoso-Trabucco A, GarcíaRupaya C. Resistencia de unión a la dentina de dos cementos selladores de endodoncia después de la medicación intraconducto de hidróxido de calcio con dos tipos de vehículos. Rev Cient Odontol (Lima). 2020; 8 (2): $\mathrm{e} 015$.

DOI: $10.21142 / 2523-2754-0802-2020-015$

\section{RESUMEN}

Objetivo: Comparar la resistencia de unión a la dentina del cemento sellador tipo Grossman (Grossdent) y Apexit Plus (Ivoclar Vivadent) después de la medicación intraconducto de hidróxido de calcio $\mathrm{Ca}(\mathrm{OH})^{2}$ con vehículos de paramonoclorofenol alcanforado (PMCFA) y de clorhexidina al 2\% (CHX). Metodología: Estudio experimental in vitro. Se seleccionó una muestra de 44 dientes humanos monorradiculares y fueron divididos en 4 grupos con medicación intraconducto $(n=9)$ y 2 grupos control. Los dientes fueron desinfectados y los conductos radiculares instrumentados usando una técnica híbrida. Se medicaron dos grupos con hidróxido de calcio más PMCFA y dos grupos con hidróxido de calcio más CHX 2\% durante dos semanas. De cada grupo con medicación uno fue obturado con cemento sellador endodóntico Grossman y el otro con Apexit Plus, mediante la técnica de compactación lateral. Los grupos control fueron obturados solo con los dos cementos anteriormente mencionados. Se cortaron las muestras en discos de $2 \mathrm{~mm}$ de espesor y fueron sometidas a prueba de empuje con una máquina de ensayo universal. Resultados: El valor de la media más alta lo obtuvo el grupo medicado con $\mathrm{Ca}(\mathrm{OH})^{2}$ más PMCFA y obturado con Apexit Plus (m 3,742 y DE 0,808), mientras que el valor más bajo lo presentó el grupo de $\mathrm{Ca}(\mathrm{OH})^{2}$ más PMCFA obturado con cemento Grossman (m 1,371 y DE 0,699), presentaron diferencias significativas $(\mathrm{p}<0,001)$. Conclusión: La resistencia de unión a la dentina radicular se ve influenciada por la medicación intraconducto previa a la obturación endodóntica.

Palabras clave: medicación intraconducto, hidróxido de calcio, resistencia de unión

\begin{abstract}
Objective: Compare the dentin bond strength of Grossman's sealer (Grossdent) and Apexit Plus sealer (IvoclarVivadent) after intra-canal medication of calcium hydroxide $\mathrm{Ca}(\mathrm{OH})^{2}$ associated with vehicles of camphorated parachlorophenol (CMPC) and chlorhexidine 2\% (CHX). Methodology: This was an in vitro experimental study. A sample of 44 monoradicular human teeth were disinfected and divided into 4 groups of intra-canal medication $(\mathrm{N}=9)$ and 2 control groups. The teeth were disinfected and root canals were instrumented using a hybrid technique. Two groups were medicated with $\mathrm{Ca}(\mathrm{OH})^{2}$ and $\mathrm{CMPC}$ and two groups with $\mathrm{Ca}(\mathrm{OH})^{2}$ and $\mathrm{CHX}$ $2 \%$ for two weeks. One group of each medication was filled with Grossman's sealer and the others with Apexit Plus sealer using the lateral compaction technique. The control groups were filled only with the two sealers mentioned previously. Samples were cut into $2 \mathrm{~mm}$ thick discs and placed on a push test with a universal testing machine. Results: The highest value of the means was obtained by the group medicated with $\mathrm{Ca}(\mathrm{OH})^{2}$ in association with $\mathrm{CMPC}$ and filled with Apexit Plus (m 3.742; SD 0.808), while the lowest value was presented by the group of $\mathrm{Ca}(\mathrm{OH})^{2}$ associated with CMPC, filled with Grossman's sealer (m 1,371; SD 0.699). Statistical differences were considered with $\mathrm{p}<0.001$. Conclusion: Root dentin bond strength is influenced by intra-canal medication prior to endodontic filling.
\end{abstract}

Keywords: intra-canal medication, calcium hydroxide, bond strength

\footnotetext{
1 Residente Especialidad en Carielogía y Endodoncia, Universidad Científica del Sur. Lima, Perú

2 Docente Especialidad en Carielogía y Endodoncia, Universidad Científica del Sur. Lima, Perú
} 


\section{INTRODUCCIÓN}

Uno de los objetivos primordiales en el tratamiento de patologías pulpares y periapicales es lograr la eliminación de los microorganismos existentes en el sistema de conductos radiculares presente en los dientes. Este propósito se puede cumplir mediante la combinación de una buena instrumentación, abundante irrigación de los conductos radiculares para eliminar el smear layer (1, $\left.{ }^{2}\right)$ y diversas técnicas de tratamiento de acuerdo con el diagnóstico pulpo-periapical.

En algunos casos, el uso de medicamentos dentro del sistema de conductos es recomendado para aumentar la desinfección de los conductos radiculares, especialmente en lugares poco accesibles a la instrumentación, a fin de inhibir el crecimiento bacteriano y potenciar la reparación periapical de la pieza tratada.

Uno de los medicamentos intraconducto ampliamente utilizado y aceptado por sus cualidades es el hidróxido de calcio, el cual debe eliminarse antes de llenar los conductos radiculares; sin embargo, su remoción completa es problemática y parte de él puede retenerse en el área apical $(3,4)$. Los residuos de hidróxido de calcio utilizados como medicación intraconducto, que probablemente permanecen en la entrada de los túbulos y en las paredes dentinales, pueden afectar la penetración del cemento endodóntico en los túbulos dentinarios e impedir el correcto sellado hermético $(5,6)$.

La resistencia de unión a la dentina intrarradicular de materiales endodónticos indica la fuerza con la que el material de obturación está pegado en las paredes dentinarias. Esta resistencia previene la fuga coronal y apical del material, y apoya al tejido dental contra la fractura radicular durante las maniobras de llenado de la raíz. La prueba de expulsión de material intrarradicular es frecuentemente usada para evaluar la efectividad de materiales dentales en la dentina del conducto radicular, las pruebas con valores altos contribuyen a la longevidad y a un buen pronóstico en los tratamientos endodónticos. La resistencia de unión depende, entre otros, de los materiales usados durante el tratamiento de conductos.
Existen diferentes vehículos con los cuales podemos transportar el hidróxido de calcio en polvo, como la solución salina, el agua destilada, el líquido anestésico y los utilizados en este estudio: la clorhexidina al $2 \%$ y el paramonoclorofenol alcanforado $\left({ }^{11}\right)$.

La clorhexidina es un agente antibacteriano de amplio espectro usado durante el proceso de preparación endodóntica y como irrigante final. Tiene actividad antimicrobiana contra una amplia gama de microorganismos orales, como las bacterias gram positivas y las gram negativas, $y$ una alta sustantividad $y$ capacidad de inhibir las metaloproteinasas de la matriz dentinaria. También es usado como medicamento intraconducto, solo o combinado con otras sustancias para potenciar su acción $\left({ }^{7-10}\right)$.

Otra sustancia ampliamente manejada como medicación intraconducto es el paramonoclorofenol alcanforado. Este es un antiséptico intraconducto oleoso muy utilizado, de color ámbar y baja tensión superficial. Su acción antibacteriana se debe a los dos radicales que lo componen, el fenol y el cloro. Asimismo, la asociación del paramonoclorofenol con el alcanfor reduce su acción irritante, debido a que causa una liberación más lenta de dicha sustancia, lo cual disminuye su poder de agresión a los tejidos. Es utilizado para tratamientos en dientes necróticos y ha sido estudiado en diversos artículos por su rápida acción bactericida $\left({ }^{12}\right)$.

El propósito del presente estudio fue determinar la resistencia de unión a la dentina en conductos previamente medicados con hidróxido de calcio con dos vehículos, cuando se utiliza un sellador de óxido de zinc y eugenol, y otro de hidróxido de calcio.

\section{MATERIALES Y MÉTODOS}

El presente estudio fue aprobado por el Comité de Ética de la Universidad Científica del Sur, con número de aprobación 646. Para el cálculo del tamaño muestral se empleó la fórmula para comparar medias con un nivel de confianza del $95 \%$ y con una precisión del $5 \%$, y se obtuvo un total de 9 muestras por grupo $(n=44)$, distribuidas en 
cuatro grupos según el medicamento asignado $(n=9)$ y dos grupos control sin medicamento intraconducto. Los criterios de inclusión comprendían dientes humanos monorradiculares de un solo conducto sin grietas, caries, reabsorción o restauraciones radiculares.

Se utilizó un disco de corte diamante ultrafino C03/190 (China) de baja velocidad para remover las coronas de los dientes unirradiculares y se dejó a todos con una longitud radicular de $10 \mathrm{~mm}$ (figuras 1 y 2).

Una lima K-Flexofile de \#15 (Densply, Maillefer) se utilizó para determinar la longitud de trabajo. La lima fue puesta en el conducto radicular y, cuando estuvo visible en el foramen apical, se redujo $1 \mathrm{~mm}$ y se tomó la longitud de trabajo $\left({ }^{13}\right)$. Los conductos radiculares fueron preparados usando una técnica híbrida por la cual primero se utilizó la lima rotatoria Introfile para ensanchar el tercio coronal y así estandarizar la entrada de los conductos. Luego, se utilizaron limas manuales para trabajar el tercio apical de cada conducto desde la lima \#15 hasta la \#40 K-Flexofile. Finalmente, se emplearon limas de segunda serie, desde la lima \#45 hasta la lima \#70 K-File, para reducir un milímetro consecutivamente en sentido coronal.

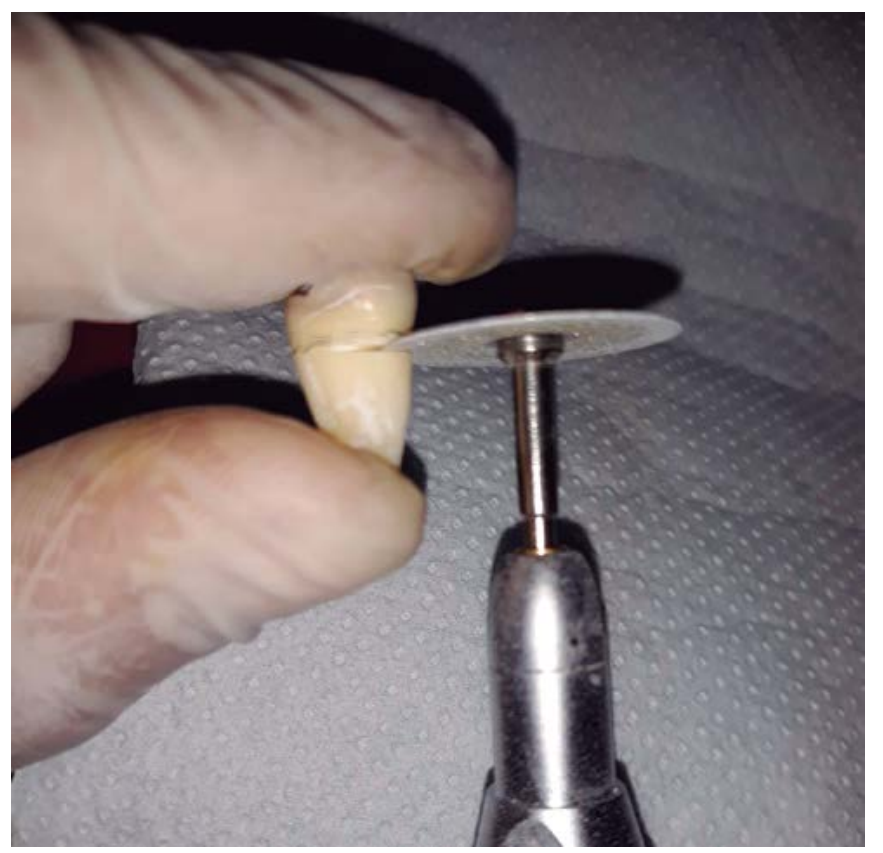

Figura I. Corte de piezas dentarias.
Se utilizaron aproximadamente $2 \mathrm{ml}$ de $\mathrm{NaOCl}$ al 2,5\% para irrigar cada muestra mediante una jeringa con punta NaviTip (Ultradent). Posteriormente, el conducto radicular se irrigó con $2 \mathrm{ml}$ de EDTA (Maquira, Brasil) durante 3 minutos y se enjuagó con $5 \mathrm{ml}$ de agua destilada. Los canales radiculares fueron secados con puntas de papel y luego se los dividió en grupos.

Se medicaron 18 muestras del total con aproximadamente 0,05 $\mathrm{ml}$ de la mezcla obtenida de 0,04 ml de hidróxido de calcio con $0,01 \mathrm{ml}$ de paramonoclorofenol alcanforado, y otras 18 con aproximadamente $0,05 \mathrm{ml}$ de la mezcla obtenida de 0,05 $\mathrm{ml}$ de hidróxido de calcio con 0,01 $\mathrm{ml}$ de clorhexidina al $2 \%$, transportados mediante una jeringa con cánula de aplicación tipo 41 (VOCO), y se mantuvieron almacenados durante dos semanas.

El acceso coronal fue sellado con Coltosol F (Coltene) y almacenado a temperatura ambiente durante dos semanas. Después de este tiempo, los medicamentos intraconducto fueron retirados mediante irrigación con $5 \mathrm{ml}$ de agua destilada alternando con una lima \#40 K-Flexofile a longitud de trabajo, y se irrigó finalmente con $5 \mathrm{ml}$ de agua destilada $\left({ }^{13}\right)$.

Cada grupo medicado fue subdividido en dos subgrupos y cada muestra fue rotulada con la letra correspondiente:

- Subgrupo A: medicación con hidróxido de calcio con paramonoclorofenol alcanforado / cemento sellador endodóntico Grossman.

- Subgrupo B: medicación con hidróxido de calcio con paramonoclorofenol alcanforado / cemento sellador endodóntico Apexit Plus.

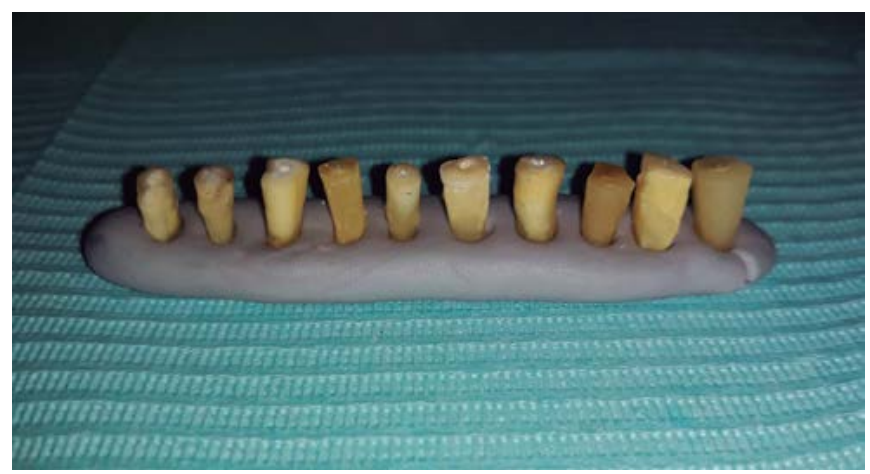

Figura 2. Piezas dentarias con una longitud de $10 \mathrm{~mm}$. 
- Subgrupo C: medicación con hidróxido de calcio con clorhexidina al $2 \%$ /cemento sellador endodóntico Grossman.

- Subgrupo D: medicación con hidróxido de calcio con clorhexidina al $2 \%$ / cemento sellador endodóntico Apexit Plus.

Todos los subgrupos fueron obturados con conos de gutapercha n. 40 y accesorios, mediante la técnica de compactación lateral.

El tiempo de espatulado del cemento sellador Grossman en polvo (Grossdent) y líquido (Eugenol) fue de aproximadamente $1 \mathrm{~min}$ y el cemento sellador Apexit Plus (Ivoclar Vivadent) fue colocado mediante la punta mezcladora del mismo producto, obturados con conos accesorios de gutapercha de primera serie y compactador digital A y B (Dentsply, Maillefer) de 21 mm (figura 3).

Todas las muestras fueron selladas con Coltosol y almacenadas durante dos semanas a temperatura ambiente en una caja separadora diferenciada y rotulada con los cuatro grupos medicados y los dos grupos control. Luego, se cortaron las raíces con $2 \mathrm{~mm}$ de espesor en el tercio medio de cada muestra utilizando un disco de corte diamante ultrafino C03/190 (China) de baja velocidad, con irrigación copiosa de agua (figura 4).

Cada raíz se sometió a una prueba de empuje con una máquina digital de ensayo universal certificada (Inmelab: Ingeniería y Metrología, Perú). La fuerza de empuje fue ejercida con un pistón cilíndrico de $0,7 \mathrm{~mm}$ de diámetro, diseñado especialmente para las muestras del estudio, a una velocidad de corte de $1 \mathrm{~mm} / \mathrm{min}$, perpendicular a la superficie de la muestra (figura 5).

La máxima fuerza donde el material fue desplazado se obtuvo en Newton y convirtió en MPa usando la siguiente fórmula: área superficial bajo carga $=$ grosor de la dentina en la rebanada $\times$ la circunferencia del conducto.

Los datos de cada grupo fueron recolectados en una ficha digital para ser procesados y analizados con el programa IBM SPSS Statistics 22. Los datos no presentaron normalidad, por lo cual se utilizaron pruebas no paramétricas. En primer lugar, se realizó una estadística descriptiva y se obtuvo media, desviación estándar y valor mayor y menor. Posteriormente, se realizó una estadística inferencial mediante las pruebas Kruskall Wallis para efectuar las comparaciones entre todos los grupos y, luego, la U Mann Whitney para comparar cada dos grupos.

\section{RESULTADOS}

En la tabla 1 se observa que el valor de la media más alta lo obtuvo el grupo de paramonoclorofenol alcanforado y

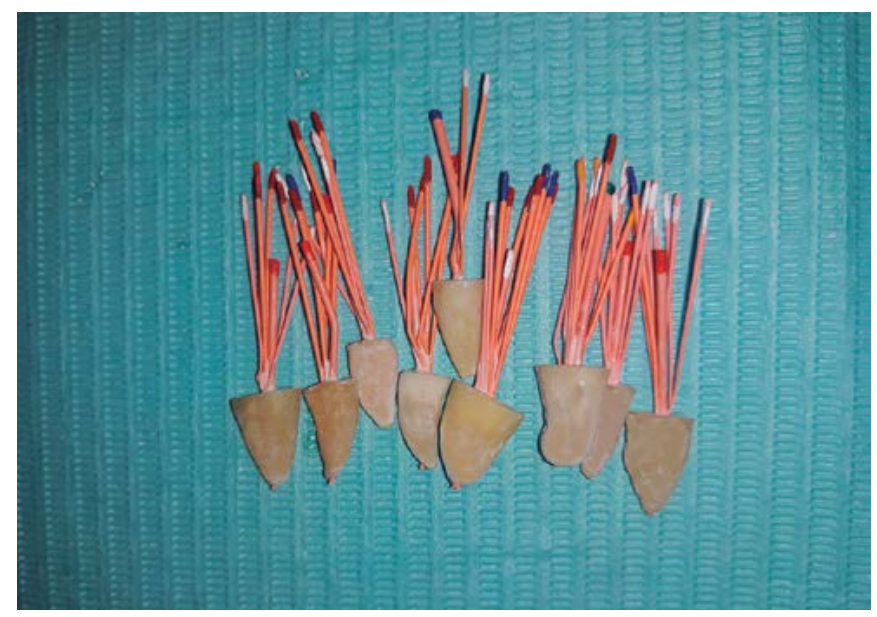

Figura 3. Piezas dentarias obturadas con cemento sellador endodóntico y conos de gutapercha.

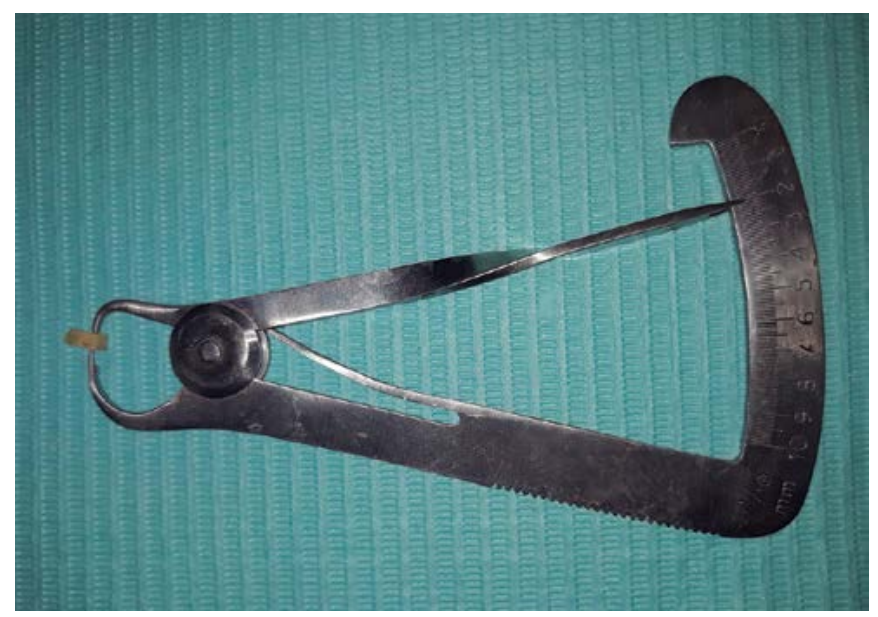

Figura 4. Raíces calibradas a $2 \mathrm{~mm}$ de espesor. 


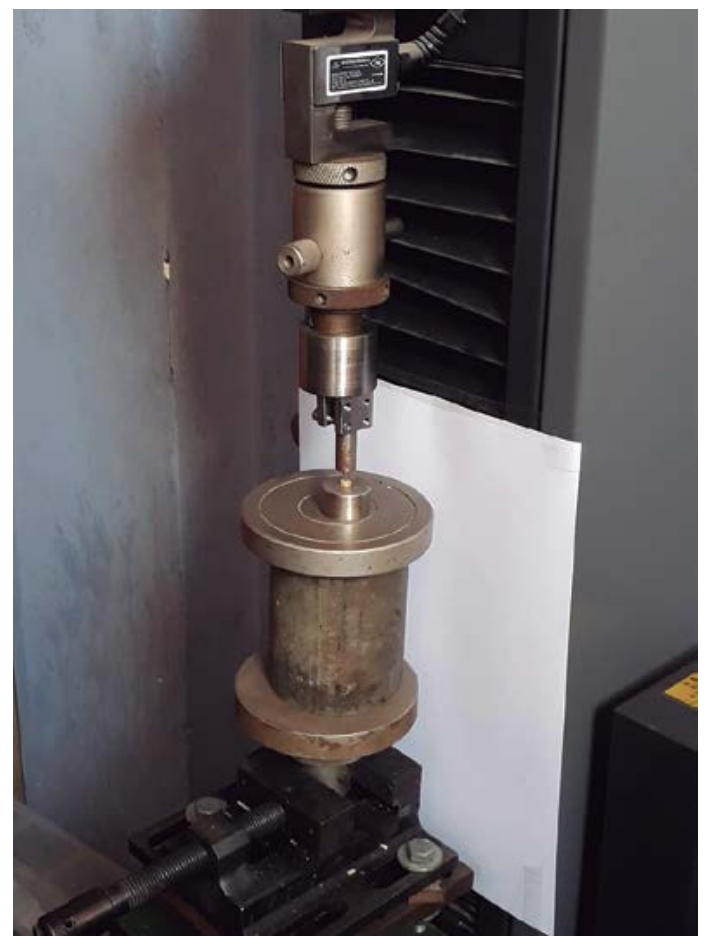

Figura 5. Prueba de empuje con máquina digital de ensayo universal

Tabla 1. Descripción de los valores de la resistencia de unión a la dentina (MPa) de dos cementos selladores en dientes previamente medicados con hidróxido de calcio con dos vehículos

\begin{tabular}{|c|c|c|c|c|c|}
\hline Grupos & $\mathbf{N}$ & m $\mathrm{MPa}$ & DE & $\begin{array}{l}\text { Valor } \\
\text { mayor }\end{array}$ & $\begin{array}{l}\text { Valor } \\
\text { menor }\end{array}$ \\
\hline $\begin{array}{l}\text { Paramonoclorofenol alcanforado } \\
\text { y Apexit Plus }\end{array}$ & 9 & 3,742 & 0,808 & 5,07 & 2,86 \\
\hline $\begin{array}{l}\text { Paramonoclorofenol } \\
\text { alcanforado y Grossman }\end{array}$ & 9 & ।,37। & 0,699 & 2,51 & 0,42 \\
\hline Clorhexidina al $2 \%$ y Apexit Plus & 9 & 2,989 & 0,523 & 3,76 & 2,43 \\
\hline Clorhexidina al $2 \%$ y Grossman & 9 & 2,819 & 0,687 & 3,82 & 1,89 \\
\hline Control Apexit Plus & 4 & 2,103 & 1,111 & 3,56 & 0,89 \\
\hline Control Grossman & 4 & ।,785 & 0,876 & 2,46 & 0,59 \\
\hline
\end{tabular}

Prueba: Kruskall Wallis

$p<0,001$
Apexit Plus, y fue de 3,742 con una desviación estándar de 0,808 , mientras que el valor más bajo lo presentó el grupo de paramonoclorofenol alcanforado y Grossman, con una media de 1,371 y una desviación estándar de 0,699. Al aplicar la prueba de Kruskall Wallis se encontró que existe una diferencia estadísticamente significativa $(\mathrm{p}<0,001)$.

En la comparación de pares entre los grupos de estudio, se encontraron diferencias estadísticamente significativas al aplicar la prueba U de Mann Whitney (tabla 2).

\section{DISCUSIÓN}

El material ideal de relleno del canal radicular debe adherirse a las paredes dentinarias y resistir fuerzas dislocantes. Para lograr esto, el sellador debe unirse tanto como sea posible a la dentina del conducto radicular y los pasos previos a la obturación de los conductos deben ser controlados y bien ejecutados.

El presente estudio comparó la resistencia de unión a la dentina de dos cementos selladores endodónticos en presencia de diferentes medicamentos intraconducto. Se encontró que el cemento Apexit Plus presenta mayor resistencia de unión a la dentina que el cemento Grossman al medicar previamente los conductos con paramonoclorofenol alcanforado. Esto contradice a Harikumar (2014) que, al realizar pruebas para medir la fuerza de unión de los selladores, reveló que los selladores a base de hidróxido de calcio tienen menos adherencia a la dentina que los selladores a base de óxido de zinc y eugenol, y que al agregar amoxicilina como medicamento estos resultados no fueron afectados $\left({ }^{14}\right)$.

Tabla 2. Comparación de la resistencia de unión (valor p) de los cementos selladores cada dos grupos de prueba.

\begin{tabular}{lcccc}
\multicolumn{1}{c}{ Grupos } & $\begin{array}{c}\text { Paramonoclorofenol } \\
\text { alcanforado y } \\
\text { Grossman }\end{array}$ & $\begin{array}{c}\text { Clorhexidina al 2\% } \\
\text { y Apexit Plus }\end{array}$ & $\begin{array}{c}\text { Clorhexidina al 2\% } \\
\text { y Grossman }\end{array}$ & $\begin{array}{c}\text { Control Apexit } \\
\text { Plus }\end{array}$ \\
\hline Paramonoclorofenol alcanforado y Apexit Plus & $<0,001$ & 0,050 & 0,031 & 0,050 \\
Grossmantrol
\end{tabular}


Se ha recomendado el uso de medicamentos intraconducto entre las sesiones de tratamiento para promover la desinfección dentro del conducto durante los procedimientos de endodoncia $(14,15)$. Algunos estudios han demostrado que los irrigantes químicos y los medicamentos intraconducto pueden afectar las propiedades de la dentina radicular en la interfaz de esta y el material de obturación, y que el efecto de los medicamentos intraconducto en la fuerza de la unión entre el sellador y la dentina parece ser importante debido a la penetración del cemento obturador en los túbulos dentinarios (2, 3, 16). Margelos, Eliades, Calt, Serper, Kim y Kim demostraron que la existencia de hidróxido de calcio en las paredes del conducto puede afectar la penetración de los cementos selladores en los túbulos dentinarios $\left({ }^{4,17,18}\right)$.

En el presente estudio, los resultados muestran que sí existen diferencias significativas en la fuerza de unión a la dentina $(p<0,001)$. El grupo medicado con paramonoclorofenol alcanforado y obturado con Apexit Plus tuvo la mayor resistencia de unión de todos los grupos, seguido por el grupo con el mismo cemento obturador previa medicación con clorhexidina al $2 \%$.

Los resultados muestran que el cemento Apexit Plus tiene mejor adherencia a la dentina radicular en cualquiera de los grupos, lo cual concuerda con Sifuentes, quien refiere que, al realizar una evaluación del sellado a nivel apical usando un microscopio electrónica de barrido, la capacidad de sellado del cemento Apexit fue mayor que la del cemento Grossman.

Se estima que la técnica de irrigación utilizada en el sistema de conductos radiculares tiene un papel muy importante, ya que elimina el hidróxido de calcio, aunque deja presencia residual, por lo que la resistencia de unión se ve afectada en cierto rango. Se ha reportado que, después del uso de ciertos medicamentos intraconducto, es posible observar sus remanentes en el $45 \%$ de las paredes del conducto, incluso después de los intentos por eliminarlo. Los residuos de hidróxido de calcio en los conductos radiculares pueden generar una capa gruesa y no homogénea de cemento endodóntico y también ocasionar una reacción química con el cemento endodóntico que resulta en una reducción del tiempo de trabajo y puede afectar la penetración de los selladores en los túbulos dentinarios $\left({ }^{19}\right)$.

El protocolo de irrigación en el presente estudio fue tomado del artículo base "Efectos de diferentes medicamentos intraconducto en la resistencia de unión de los cementos endodónticos» $\left.{ }^{(13}\right)$, donde la irrigación final después de la eliminación de medicamentos en los conductos se realizó con agua destilada. El uso de EDTA como solución irrigante final no se empleó para comprobar los efectos de la medicación intraconducto y que dicha solución quelante no interfiera o altere los resultados de las muestras. Asimismo, los protocolos de irrigación pueden variar según el investigador y podrían ser materia de próximas investigaciones.

En el marco de futuras investigaciones referentes a la fuerza de unión y los materiales endodónticos, el estudio de los nuevos biocerámicos y los medicamentos intraconducto sería un gran aporte para la práctica clínica en endodoncia, ya que de este trabajo se concluye que la resistencia de unión a la dentina radicular sí se ve influenciada por los tratamientos de medicación previos a la obturación.

\section{CONCLUSIÓN}

Se concluye que el grupo medicado con hidróxido de calcio más paramonoclorofenol alcanforado y obturado con cemento Apexit Plus tuvo mejores resultados en la resistencia de unión a la dentina.

Contribución de los autores: Angela Carolina Reinoso Trabucco ha participado en la concepción, la recolección de información, el análisis e interpretación de datos, la redacción y la aprobación de la versión final del artículo. Carmen Rosa García Rupaya ha participado en la concepción, el análisis e interpretación de datos, la redacción y la aprobación de la versión final del artículo.

Conflicto de intereses: Las autoras declaran no tener conflictos de intereses de ningún tipo.

Fuentes de financiamiento: Autofinanciado. 


\section{REFERENCIAS BIBLIOGRÁFICAS}

1. Pérez D, Pineda V, Plaza M, Quiñónez A, Pineda H, Orozco Y, et al. Cementos endodónticos. Ciudad de Guatemala: Universidad de San Carlos de Guatemala; 2012.

2. Alvear J, Pupo S, Flórez JE, Díaz A, Pérez L, Velásquez A. Evaluación de la penetración de cementos obturadores de canales mediante microscopía electrónica de barrido. Av Odontoestomatol. 2017; 33 (4): 143-9.

3. Carvalho CN, Bauer J, Ferrari PH, Souza SF, Soares SP, Loguercio $\mathrm{AD}$, et al. Influence of calcium hydroxide intracanal medication on bond strength of two endodontic resin-based sealers assessed by micropush-out test. Dent Traumatol. 2013; 29: 73-6. doi: 10.1111/j.1600-9657.2011.01109.x

4. Kim S, Kim Y. Influence of calcium hydroxide intracanal medication on apical seal. Int Endod J. 2020; 35 (7): 623-8. doi: 10.1046/j.1365-2591.2002.00539.x

5. Farhad A, Barekatain B, Allameh M, Narimani T. Evaluation of the antibacterial effect of calcium hydroxide in combination with three different vehicles: An in vitro study. Dent Res J. 2012; 9 (2): 167-72. doi: 10.4103/1735-3327.95231

6. Sahebi S, Moazami F, Abbott P. The effects of short-term calcium hydroxide application on the strength of dentine. Dent Traumatol 2010; 26: 43-6. doi: 10.1111/j.1600-9657.2009.00834.x

7. Gomes B, Vianna M, Sena N, Zaia A, Ferraz C, de Souza Filho F. In vitro evaluation of the antimicrobial activity of calcium hydroxide combined with chlorhexidine gel used as intracanal medicament. Oral Surg Oral Med Oral Pathol Oral Radiol Endod. 2006; 102: 544-50. doi: 10.1016/j.tripleo.2006.04.010

8. Valera M, Salvia A, Maekawa L. Antimicrobial analysis of chlorhexidine gel and intracanal medicaments against microorganisms inoculated in root canals. Minerva Stomatol. 2010; 59 (7-8): 415-21.

9. Gondim J, Avaca-Crusca J, Valentini S, Zanelli C, Spolidorio D, Giro E. Effect of a calcium hydroxide/chlorhexidine paste as intracanal dressing in human primary teeth with necrotic pulp against Porphyromonas gingivalis and Enterococcus faecalis. Int J Paediatr Dent. 2012; 22 (2): 116-24. doi: 10.1111/j.1365263X.2011.01174.x

10. Gomes B, Vianna M, Zaia A, Almeida J, de Souza-Filho F, Ferraz C. Chlorhexidine in endodontics. Braz Dent J. 2013; 24 (2): 89-102. doi: 10.1590/0103-6440201302188
11. Zumárraga M, Almeida A, Resende da Silva T, Henrique $\mathrm{P}$, Ferreira da Silva G. Beneficio de algunas asociaciones como medicación intracanal. KIRU. 2017; 14 (2): 188-92. doi: https:// doi.org/10.24265/kiru.2017.v14n2.12

12. Peñaloza U, Condori-Condori G. Efecto antibacteriano del paramonoclorofenol alcanforado vs. la asociación de hidróxido de calcio paramonoclorofenol alcanforado, sobre el cultivo in vitro de Enterococcus faecalis. Rev Med Basadrina 2016; 10 (1): 16-9. doi: https://doi.org/10.33326/26176068.2016.1.590

13. Shakouie S, Shahi S, Samiei M, Milani A, Reyhani M, Paksefat $\mathrm{S}$, et al. Effects of different intra canal medicaments on the push out bond strength of endodontic sealers. J Clin Exp Dent. 2017; 9 (3): e443-7. doi: $10.4317 /$ jced.53522

14. Harikumar V, Ravichandra P, Jayaprada S, Ramkiran D, Jaya M, Rajani S. Comparative evaluation of push-out bond strength of three endodontic sealers with and without amoxicillin - An invitro study. J Clin Diagn Res. 2014; 8 (1): 228-31. doi: 10.7860/ JCDR/2014/7180.3919

15. Ercan E, Dalli M, Turksel D, Yaman F. Effect of intracanal medication with calcium hydroxyde and $1 \%$ chlorhexidine in endodontic retreatment cases with periapical lesions: An in vivo study. J Formos Med Assoc. 2007; 106 (3): 217-24. doi: 10.1016/ s0929-6646(09)60243-6

16. Athanassiadis B, Abbott P, Walsh L. The use of calcium hydroxide, antibiotics and biocides as antimicrobial medicaments in endodontics. Aust Dent J. 2007; 52 (1): 64-82. doi: 10.1111/ j.1834-7819.2007.tb00527.x

17. Mishra P, Sharma A, Mishra S, Gupta M. Push-out bond strength of different endodontic obturation material at three different sites - In-vitro study. J Clin Exp Dent. 2017; 9 (6): e733-e737. Published 2017 Jun 1. doi: 10.4317/jced.53647

18. Margelos J, Eliades G, Verdalis C, Palaghias G. Interaction of calcium hydroxide with zinc oxide eugenol type sealers: a potential clinical problema. J Endod. 1997; 23: 43-8. doi: 10.1016/S00992399(97)80206-3

19. Calt S, Serper A. Dentinal tubule penetration of root canal sealers after root canal dressing with calcium hydroxide. J Endod. 1999; 25: 431-3. doi: 10.1016/S0099-2399(99)80273-8 